Ann. Biol. anim., Bioch., Biophys., 1965, 5 (3), 403-406

\title{
NOTE PRÉLIMINAIRE SUR UN LOCUS SUPPLÉMENTAIRE DE GROUPES SANGUINS DES BOVINS, LE LOCUS $\mathbf{T}^{\prime}$
}

\author{
F. GROSCLAUDE \\ avec la collaboration technique de Anne LE Grand, \\ G. Houlier, G. Ruffet \\ Station centrale de Génétique animale, \\ Centre national de Recherches zootechniques, Jouy-en-Josas (Seine-et-Oise)
}

SOMMAIRE

Un nouvel isoimmunanticorps bovin, obtenu à deux reprises, détecte un facteur antigénique qui n'est déterminé par aucun des dix loci de groupes sanguins actuellement connus. Cet anticorps révèle donc l'existence d'un "nouveau " locus, dénommé $T$ '. La recherche de liaisons génétiques éven. tuelles entre le locus $\mathrm{T}^{\prime}$ et les autres loci est en cours. La fréquence du facteur $\mathrm{T}^{\prime}$ varie selon les races.

\section{INTRODUCTION}

On a décrit jusqu'à présent dix loci contrôlant le polymorphisme antigénique des hématies des bovins ; ce sont les loci A, B, C, FV, J, L, M, S, Z et R' S'. I'existence d'un onzième locus, le locus $N$, avait été brièvement signalée aux Etats-Unis (MILLER, I96I), mais, selon STORMont (r964), les réserves d'anti-N sont épuisées, et les essais de reproduction de cet anticorps ont échoué. On ne peut donc plus, à l'heure actuelle, faire état de ce locus.

La présente note a pour but de signaler la mise en évidence d'un onzième locus de groupes sanguins des bovins, que nous avons appelé ' $\mathrm{T}$ ' en suivant le principe de la nomenclature utilisée jusqu'ici.

\section{MÉTHODES ET RÉSULTATS}

\section{Obtention et propriétés de l'anticorps}

L'anticorps anti-T' a été obtenu une première fois par isoimmunisation d'un bovin de race Normande $\left(\mathrm{n}^{\circ} 235^{8}\right)$. Son titre est de l'ordre de $\mathrm{I} / 3^{2}$, et son affinité bonne, puisque l'hémolyse complète des échantillons positifs est en général réalisée trente minutes à une heure après la distribution du 
complément ( $\left.{ }^{1}\right)$. Anti-' $\mathrm{I}^{\prime}$ a été obtenu une seconde fois, mais avec un titre de $1 / 8$ et une affinité moindre chez un autre bovin de race Charollaise $\left(\mathrm{n}^{\circ} 346 \mathrm{r}\right)$.

La première préparation d'anti-T' présente la particularité d'être absorbable par des hématies que, par ailleurs, elle n'hémolyse pas dans les conditions habituelles. Tout se passe donc comme si le test d'hémolvse d'une part, et le test d'absorption d'autre part, permettaient de définir un système triple de sous-groupes linéaires.

\section{Mode d'hérédilé du facteur ' 1 '}

Le mode d'hérédité du facteur ' $\mathrm{T}$ ' a été analysé dans la descendance de plusieurs taurcaux hétérozygotes pour ce facteur et choisis de manière à ce que chacun des dix loci de groupes sanguins se trouve également à l'état hétérozygote chez l'un au moins d'entre eux. Pour ce travail, n'ont été retenus que les produits dont on connaissait également le groupe sanguin de la mère. L'appartenance éventuelle du facteur ' $T$ ' à chacun de ces loci était alors testée séparément, en analysant la transmission relative par le père des allèles du locus considéré et du facteur T'.

A titre d'exemple, nous donnons les résultats obtenus dans la descendance d'un taureau (jui montrent que le facteur ' $\mathrm{T}$ ' n'appartient pas au locus $\mathrm{B}$ (tabl. I).

\section{TABI,EAU I}

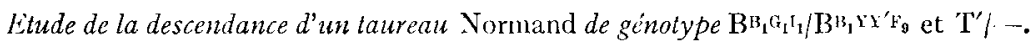

Dans cette famille, il a été possible, pour les 26 couples étudiés, de déterminer les allèles transmis par le père aux deux loci $\mathrm{B}$ et $\mathrm{T}^{\prime}$

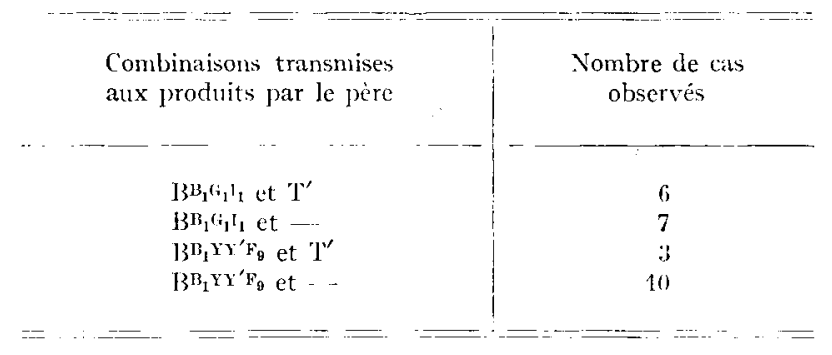

Fotons que, selon le tableau I, le facteur 'T' n'a été transmis du père aux produits que 9 fois sur 26 , au lieu de 13 en moyenne. Cependant, cet écart n'est pas statistiquement significatif au seuil de 5 p. I oo. Par ailleurs, il n'est pas observé dans d'autres familles analogues, mais moins démonstratives pour cet exposé, car d'effectif plus réduit.

Des résultats semblables ont été obtenus pour les neuf autres loci. 11 en ressort que le facteur ' ${ }^{\prime}$ est déterminé par un locus différent des loci actuellement connus, le locus 'I'. Le nombre de produits figurant dans les familles des taureaux hétérozygotes est trop faible pour déterminer si ce locus est, ou non, génétiquement indépendant des autres loci. Cependant nos données actuelles semblent indiquer que s'il y avait linkage, celui-ci ne serait pas étroit $\left({ }^{2}\right)$.

En ce qui concerne le déterminisme de "l'absorption sans hémolyse "nos données sont très limitées par suite de la relative rareté du phénomène dans les races considérées jusqu'ici. Nous avons cependant observé ce qui suit :

- Cette propriété caractérise aussi bien des hématies d'animaux adultes que d'animaux jeunes : il n'y a pas de substance antigénique $T^{\prime}$ dans le sérum qui se fixerait secondairement sur les hématies comme c'est le cas pour le locus J. dait.

- Cette propriété ne se trouve que chez des individus dont un des parents au moins la possé-

- Dans la descendance d'un taureau possédant cette propriété, la moitié des produits l'avaient hérité de leur père. $1962)$.

(1) Notre technique d'analyse a été décrite dans une précédente publication (Groscraube ct Millot,

(2) Un test de $\chi^{2}$ avec correction de Yates, appliqué aux valeurs du tableau i conduirait ì rejeter l'hypothèse d'association entre les loci $\mathrm{B}$ et $\mathrm{T}^{\prime}$. 


\section{Fréquence du facteur $T^{\prime}$}

La fréquence du facteur ' $\Gamma$ ' varie d'une race à l'autre. Elle est de l'ordre de o,ıo dans la race Normande, 0,20 dans la race Charollaise, 0,02 dans la race Frisonne et 0,20 dans la race d'Abondance, race du type Pie Rouge de l'Est.

\section{DISCUSSION}

Le fait qu'anti-T' $\mathrm{T}^{\prime}$ puisse être absorbé par des hématies qu'il n'hémolyse pas n'est pas un phénomène exceptionnel en matière de groupes sanguins des bovins. Nous avons observé un phénomène de ce genre à plusieurs reprises avec des anticorps des loci $B$ et $C$. Par exemple l'immunisation avec le facteur antigénique $R_{2}$, du locus $\mathrm{C}$, donne souvent naissance à un anticorps absorbable par tous les échantillons possédant le facteur $R_{2}$, mais n'hémolysant que les échantillons qui sont $R_{1}$. En poursuivant l'immunisation, cet anticorps peut alors acquérir la propriété d'hémolyser toutes les hématies $R_{2}$. Chaque fois que nous avons observé ce phénomène avec un anticorps de titre et d'affinité valables, bien entendu, il indiquait l'existence de sousgroupes linéaires, c'est-à-dire une subdivision allélique.

A la lumière de ces considérations, nos données sur le locus ' $T$ ' indiquent que ce locus est vraisemblablement au moins triallélique. Il doit être possible d'obtenir un jour d'autres immunsérums et notamment un réactif hémolysant tous les échantillons qui absorbent anti- $\mathrm{T}^{\prime}$ provenant du bovin $235^{8}$. Le système de sous-groupes sera donc entièrement détectable par la réaction d'hémolyse.

Récemment, l'existence possible d'un locus supplémentaire de groupes sanguins des bovins a été annoncée par les chercheurs finlandais (MAIALA, I964). La comparaison de ce locus avec le locus ' $\mathrm{T}$ ' n'a pu encore être faite.

$$
\text { Rę̧u pour publication en janvier } 1965 .
$$

\section{REMERCIEMENTS}

Yous remercions le $\mathrm{D}^{\mathrm{r}} \mathrm{B}$. LARSEN de Copenhague, qui nous a fourni des échantillons d'anti-R' et $S^{\prime}$ qui nous ont été utiles pour ce travail. Nous exprimons notre gratitude à notre ami C. BÉrANGER qui, depuis plusieurs années, nous a généreusement aidés en mettant à notre disposition un important matériel animal.

\section{SUMMARY}

THE $\mathrm{T}^{\prime}$ LOCUS, AN ADDITIONNAL LOCUS OF CATTLE RLOOD GROUPS. PRELIMINARY NOTE

A new antibody, produced twice, detects an antigenic factor which is not determined by any of the ten blood group loci known in cattle. This antibody thus discloses a "new " locus, called ' $\mathrm{T}$ ". The search for a possible genetic linkage between this locus and the other ten blood group loci is still in progress. The frequency of factor ' $\mathrm{T}^{\prime}$ varies according to the breed. 


\section{RÉFÉRENCES BIBLIOGRAPHIQUES}

Grosclaude F., Millot P., I962. Contribution à l'étude des groupes sanguins de la race bovine Montbéliarde. Ann. Biol. anim. Bioch. Biophys., 2, 185-208.

MaIAl.A K., I 964 . Applications of immunogenetics to cattle breeding problems in Finland. Journées d'Etude Féd. Europ. Zoot., Lisbonne.

Miller W. J., i96I. Evidence for two new systems of blood groups in cattle. Genetics, 46, $88_{3}$ (Abstract). Stormont C., Ig64. Communication personnelle. 